\title{
Solitary Peripheral Osteoma of the Mandible presented as Trismus
}

\author{
${ }^{1}$ Varsha Sunil Manekar, ${ }^{2}$ Vishal Dewalwar
}

\begin{abstract}
Osteoma is rare benign tumors of bone commonly seen in the maxillofacial skeleton. Peripheral osteoma is more frequent in the mandible than the maxilla. The present article is a case report of peripheral osteoma at angle of mandible below the masseter muscle. Though the lesion is benign, the reduced mouth opening caused a lot of inconvenience to the patient. The patient's primary complaint was trismus and swelling at angle of mandible. The computed tomography (CT) scan was diagnostic of calcification at the angle. The differential diagnosis includes osteoma and calcification in masseter muscle. The lesion was excised through Risdon's incision. The surgical dissection of muscle fibers revealed the attachment of the bony lesion on the outer cortex of angle. The histopathological diagnosis confirmed the diagnosis of osteoma. The location of lesion below the masseter muscle resulted in lot of inconvenience of reduced mouth opening and diagnostic difficulty. The excision was followed by physiotherapy to increase the mouth opening.
\end{abstract}

Keywords: Peripheral osteoma, Trismus, Osteoma of mandible, Fiberoptic bronchoscope, Excision of lesion.

How to cite this article: Manekar VS, Dewalwar V. Solitary Peripheral Osteoma of the Mandible presented as Trismus. Int J Head Neck Surg 2014;5(3):161-164.

Source of support: Nil

Conflict of interest: None

\section{INTRODUCTION}

Osteoma is a benign often asymptomatic neoplasm, consisting of well-differentiated matured bone. It is characterized by proliferation of either compact or cancellous bone in an endosteal or periosteal location. ${ }^{1}$ The central osteoma arises from the endosteum, the peripheral osteoma from the periosteum, and the extra-skeletal soft-tissue osteoma usually develops within the muscle. In the facial bones, both central and peripheral osteomas have been described. Peripheral type of osteoma is most common in the lower jaws, which occurs at the surface of

\footnotetext{
${ }^{1}$ Associate Professor, ${ }^{2}$ Assistant Professor

${ }^{1}$ Department of Oral and Maxillofacial Surgery, Government Dental College and Hospital, Mumbai, Maharashtra, India

${ }^{2}$ Department of Dentistry, KEM Medical College, Mumbai Maharashtra, India

Corresponding Author: Varsha Sunil Manekar, Associate Professor, Department of Oral and Maxillofacial Surgery, Government Dental College and Hospital, Mumbai, Maharashtra, India Phone: 9823077626, e-mail: varsha_manekar@yahoo.co.in
}

the cortical bone and is pedicled or sessile. Most of the osteomas occurring in the mandible are dense osteomas, and the cancellous osteoma is comparatively rare. ${ }^{2}$ Of those that have been described, the locations are normally posterior to the premolars on the lingual surface of the mandible or in the condylar region. The solitary peripheral osteoma of the mandible is uncommon, with largest case series published in the English literature demonstrated only 43 cases not related to Gardner's syndrome. ${ }^{3}$ It is mostly seen in young adults and usually remains less than $2 \mathrm{~cm}$ in size after years of slow enlargement.

The pathogenesis of peripheral osteoma is unclear. Some investigators consider it as a true neoplasm, while others classify it as a developmental anomaly. ${ }^{4}$ Possibility of a reactive mechanism, caused by trauma or infection has also been suggested. Maxillofacial osteoma associated with cutaneous sebaceous cysts, multiple supernumerary teeth and colorectal polyposis is known as Gardner's syndrome. We present a case of solitary peripheral osteoma of the mandible not associated with Gardner's syndrome.

\section{CASE REPORT}

A 22-year-old male patient reported to this college for evaluation of a slowly enlarging swelling at the lateral surface of the left ramus of mandible. Apart from facial asymmetry, the patient had a chief complaint of progressively increasing trismus and difficulty in chewing but had no pain. Preoperative interincisal distance was

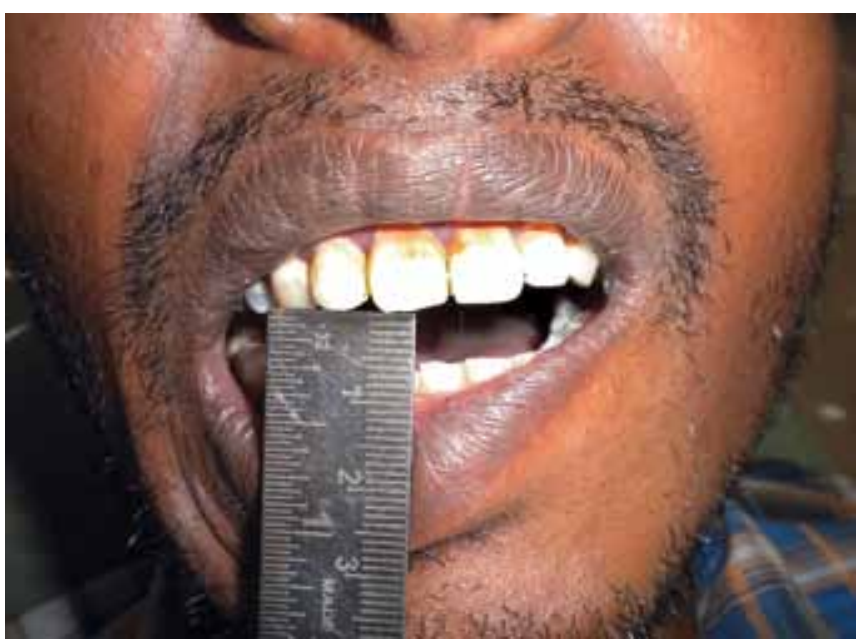

Fig. 1: Preoperative interincisal distance 
$5 \mathrm{~mm}$ (Fig. 1). Individual had no previous facial trauma or significant medical history. He had noticed the swelling for the last 1 year, and it was gradually increasing in size. There was a $5 \times 3 \mathrm{~cm}$ oval, immobile mass on the lateral surface of the left ramus of mandible. The mass was hard, nontender and nonpulsatile. The overlying skin was normal in color and showed no adhesion to the mass. There were no features of Gardner's syndrome. All laboratory findings were within normal limits. Computed tomography scan and posteroanterior (PA) mandible view showed a well circumscribed, elongated, radiopaque mass at the left ramus of mandible (Figs 2A and B).

A provisional diagnosis of peripheral osteoma was made and the patient prepared for surgery. Under general anesthesia, endotracheal intubation was done with a fiberoptic bronchoscope and the bony mass was approached through a left submandibular incision. The mass was ovoid, elongated in shape and attached to the ramus of the mandible with a narrow stalk. The lesion was divided at the stalk using a bone cutting bur and a surgical handpiece, and then completely excised using a chisel and mallet (Fig. 3A). The lateral surface of the ramus of mandible was smoothened with a vulcanite burpatient prepared for surgery. Under general anesthesia, endotracheal intubation was done with a fiberoptic bronchoscope and the bony mass was approached through a left submandibular incision. The mass was ovoid, elongated in shape and attached to the ramus of the mandible with a narrow stalk. The lesion was divided at the stalk using a bone cutting bur and a surgical handpiece, and then completely excised using a chisel and mallet (Fig. 3A). The lateral surface of the ramus of mandible was smoothened with a vulcanite bur under copious saline irrigation and the specimen (Fig. 3B) was sent for microscopic examination. Intraoperative interincisal distance was $35 \mathrm{~mm}$ and recovery was uneventful.
Histopathological examination revealed features compatible with osteoma (Fig. 4); mature cancellous bone was predominantly seen with compact bone in some sections, osteocytes and medullary spaces containing a loose connective tissue with capillaries were found. Histopathological diagnosis was 'cancellous osteoma'. Surgery was followed by rigorous physiotherapy for 1 month and postoperative interincisal distance was $25 \mathrm{~mm}$ (Fig. 5).

\section{DISCUSSION}

Osteomas are rare benign tumors of bone commonly seen in the maxillofacial skeleton. In the maxillofacial area, peripheral osteoma occurs most frequently in the sinuses. The most common site is the frontal sinus, followed by the ethmoidal and maxillary sinuses.

Peripheral osteoma has also been described in the external auditory canal, and rarely in the temporal bone and pterygoid plates. ${ }^{5}$ Peripheral osteomas are more frequent in the mandible than the maxilla. Males and females are equally affected, while the age varies between 9 and 85 years. ${ }^{6}$ These lesions usually appear as pedicled or sessile masses. The most common sites are the angle and the lower border of the body of the mandible.

The exact etiology and pathogenesis of peripheral osteoma is unknown. Both the hamartomatous and neoplastic factors have been advocated, but no definite conclusion has been reported. Infiltration of interdental bone and abnormal histological bone structure might support the neoplastic nature of this lesion. ${ }^{7}$ Developmental, neoplastic and reactive causes have been attributed as possible etiologic factors. It is unlikely that peripheral osteomas are a developmental anomaly, as most cases occur in adults. Some investigators have classified them as a reactive condition triggered by trauma, because peripheral osteomas are generally located on

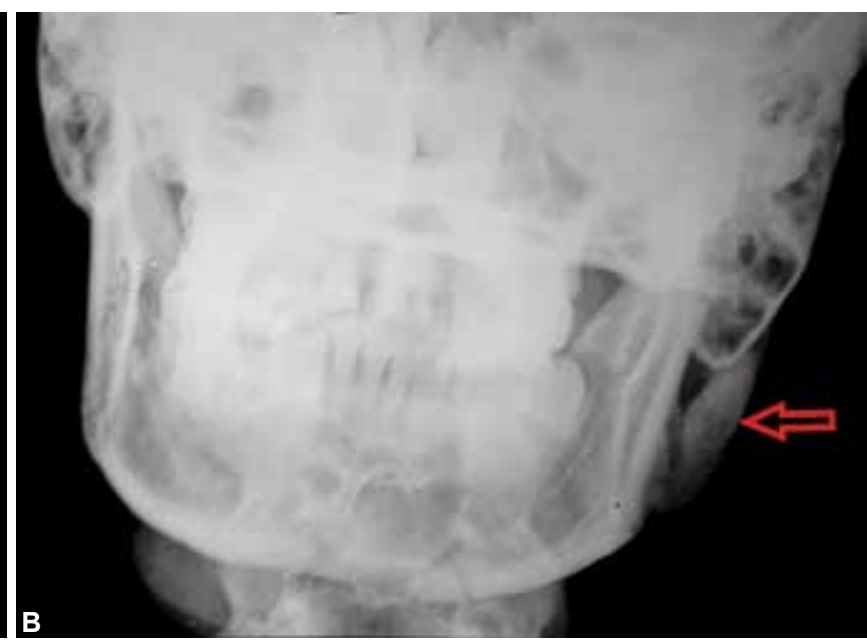

B

Figs 2A and B: (A) Computed tomography scan axial view and (B) posteroanterior mandible 

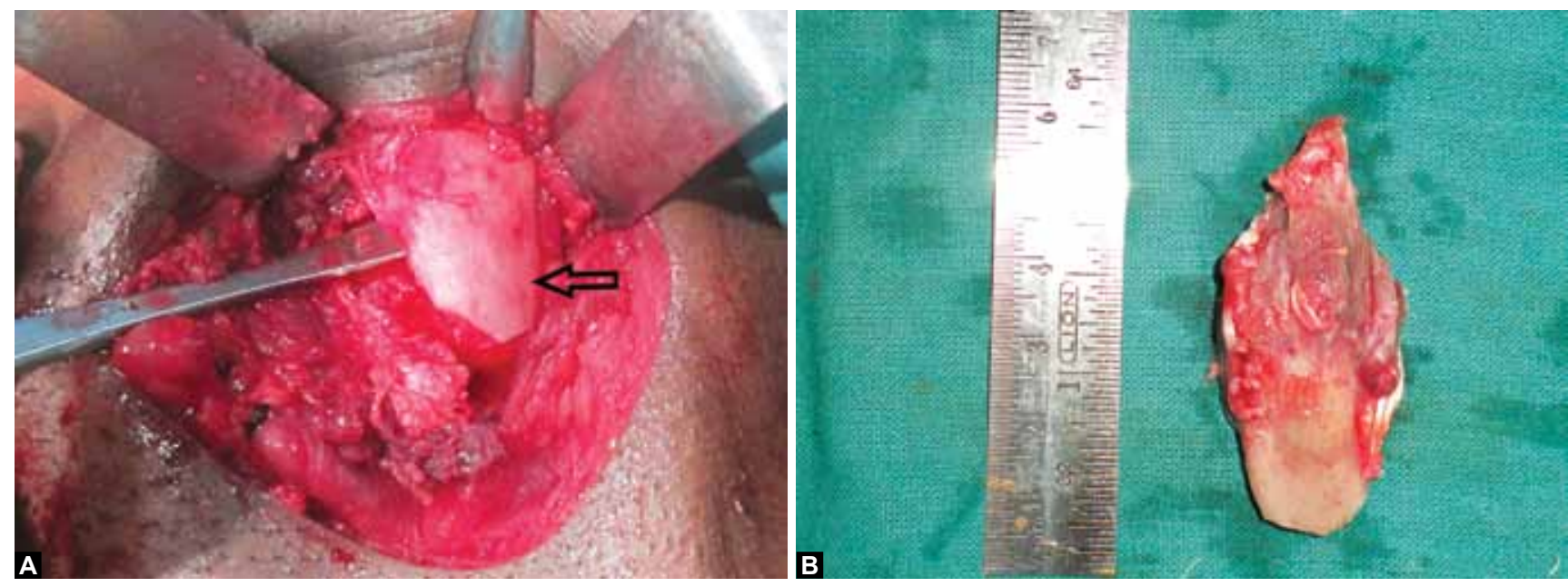

Figs 3A and B: (A) Intraoperative image showing exposed lesion and (B) specimen of excised lesion

the lower border or buccal aspect of the mandible which are areas susceptible to trauma. ${ }^{8}$ Kaplan et $\mathrm{al}^{9}$ suggested that a combination of trauma and muscle traction may play a role in its development, i.e. the trauma may cause subperiosteal bleeding or edema that would locally elevate the periosteum. This might initiate an osteogenic

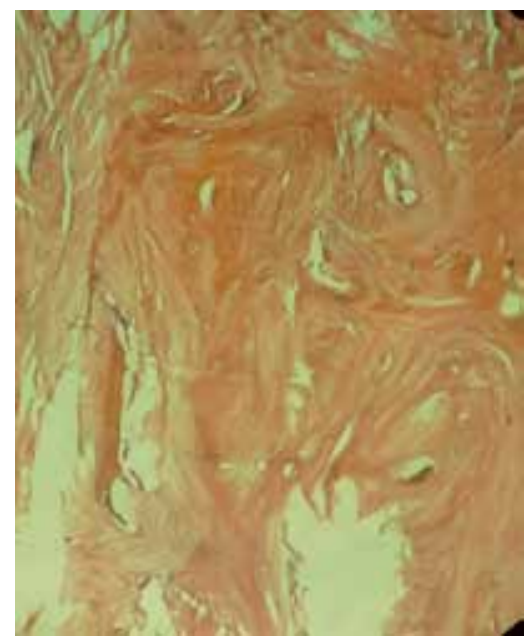

Fig. 4: Histopathology of lesion

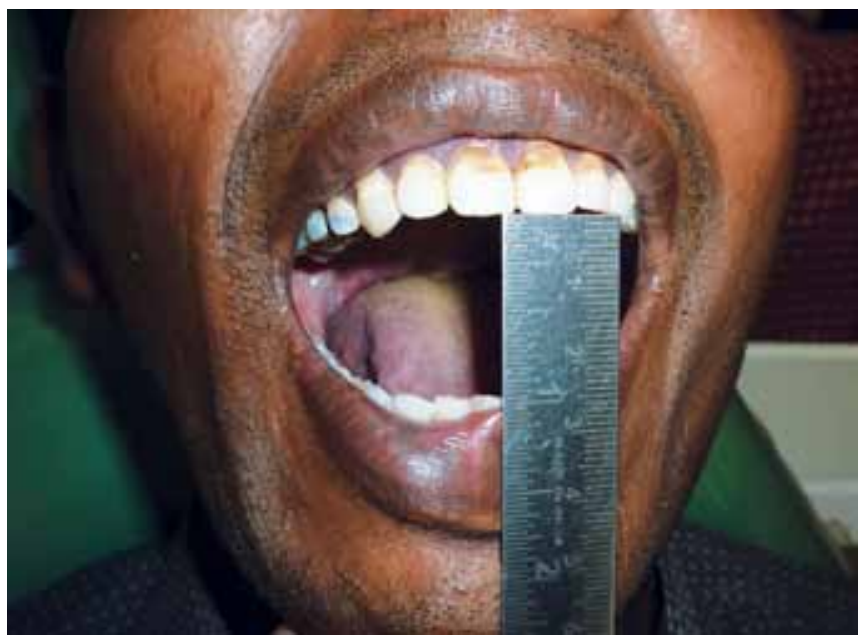

Fig. 5: Postoperative image showing increased interincisal distance reaction that could be perpetuated by continuous muscle traction. ${ }^{9}$ In our case, patient had no history of trauma; however, the mass was very close to the masseter muscle and attached to it in the inferior part which might have caused the trismus reported by the patient.

Most cases of peripheral osteoma are asymptomatic. In some, depending on the location and the size, the tumor may cause facial deformity, deviation of the mandible on opening, headache or exophthalmos. Plain radiograph (panoramic radiograph, PA mandible) or CT scan is used for imaging. Bone scan though not performed routinely, discloses the physiological activity of the peripheral osteoma.

The discovery of an osteoma of the facial skeleton should raise the possibility of Gardner's syndrome. The triad of colorectal polyposis, skeletal abnormalities and multiple impacted or supernumerary teeth is consistent with this syndrome. Skeletal involvement includes both peripheral and central osteomas. Since the osteomas often develop before the colorectal polyposis, early diagnosis of the syndrome may be life saving in certain cases. Mandibular osteomas may be a genetic marker for the development of colorectal carcinoma. ${ }^{10}$ Therefore, the patient with a diagnosis of mandibular osteoma, suspected to have Gardner's syndrome, should be further evaluated to rule out colorectal carcinoma. ${ }^{11}$ Recurrence of peripheral osteoma after surgical excision is extremely rare. Malignant transformation of peripheral osteoma has not been reported in the literature.

\section{CONCLUSION}

Osteomas are slow growing benign tumors with a very rare recurrence rate. Trismus is not a common feature of osteoma but when present special consideration should be given to the postoperative physiotherapy. Local excision is the treatment of choice. 


\section{REFERENCES}

1. Frolich Michael A. Mandibular osteoma: a case of impossible rigid laryngoscopy. J Am Soc Anesthesiolog 2000;92(1): 261-262.

2. Masuki Y. Peripheral osteoma at the mentum of mandible. Rinsho Derma 2002;44:735-737.

3. Boffano P, Roccia F, Campisi P, Gallesio C. Review of 43 osteomas of the craniomaxillofacial region. J Oral Maxillofacial Surg 2012;70(5):1093-1095.

4. Sayan NB, Cook C, Karasu HA, Gunhau O. Peripheral osteoma of the maxillofacial region: a study of 35 new cases. J Oral Maxillofac Surg 2002;60(11):1299-1301.

5. Lew D, Dewitt A, Hicks RJ, Cavalcanti MG. Osteomas of the condyle associated with Gardner's syndrome causing limited mandibular movement. J Oral Maxillofac Surg 1999;57(8): 1004-1009.

6. Johann ACBR, Fretas JB de, Aguiar MCF de, Araujo NS de, Mesquita RA. Peripheral osteoma of the mandible: case report and review of literature. J Craniomaxillofac Surg 2005;33(4): 276-281.

7. Dalambiras S, Boutsioukis C, Ioannis DMD. Peripheral osteoma of the maxilla: report of an unusual case. Oral Surg Oral Med Oral Pathol Endodontol 2005;100(1):E19-E24.

8. Kashima K, Rahman OI, Sakoda S, et al. Unusual peripheral osteoma of the mandible: report of two cases. J Oral Maxillofac Surg 2000;58(8):911-913.

9. Kaplan I, Calderon S, Buchner A. Peripheral osteoma of the mandible: a study of 10 new cases and analysis of the literature. J Oral Maxillofac Surg 1994;52(5):467.

10. Sondergaard JO, Rusmussen MS, Videbak H, Bernstein IT, Myrhoj T, Kristensen VB, et al. Mandibular osteomas in sporadic colorectal carcinoma: a genetic marker. Scad J Gastroenterol 1993;28:23-24.

11. Woldenberg Y, Nash M, Bodner L. Peripheral osteoma of the maxillofacial region-diagnosis and management: a study of 14 cases. Med Oral Pathol Oral Cir Bucal 2005;10(Suppl 2): E139-E142. 